\title{
Decoding coronary artery disease: somatic mosaicism and genomics for personal and population risk prediction
}

"By applying modern sequencing and microarray technologies in biological samples
... and by relating this integrated genome architecture-derived information to clinical
data for individual patients, we will begin to enter into the clinical genomics era."

KEYWORDS: cardiovascular disease $\|$ copy numbers " genomics $\approx$ networks $\approx$ prediction - somatic mutations

Despite advances, understanding of molecular mechanisms behind atherosclerosis and coronary artery disease (CAD) event risk prediction in individuals and the population are both still elusive. Genome-wide association studies (GWAS) have already identified a wealth of heritable point mutations such as single-nucleotide polymorphisms (SNPs) associated with blood lipid level deregulation and CAD. However, clinical utility of these disease-associated variants is modest. Furthermore, because recent evidence suggests that environmental factors causing somatic mutations accelerated during the lifetime have a more crucial role than inherited genetic variants, not only in cancer but also in other common diseases, including cardiovascular disease (CVD), current research is being reshaped. One of the crucial emerging efforts is focused on the characterization of both inherited and somatic variants. The unprecedented capacity of next-generation sequencing (NGS) and microarray technologies, to identify postzygotic mosaicism and genetic variants and to explore how these coding and noncoding sequences affect gene expression and genome function, shapes a new horizon to reach clinical implication. Here, we discuss the opportunities and challenges in discovering comprehensive diagnostics and robust prognostic tests in individuals and populations for CAD risk stratification and personalized prevention.

CVD remains the leading cause of death worldwide [101], despite substantial improvement in prevention and therapeutic strategies. Although mortality from CAD has declined over the past few decades in the industrialized world [1] due to more efficient approaches to prevent and treat, it continues to represent a major health problem [1].

Long-term mortality after myocardial infarction (MI) is still a major issue [1,2]. Left ventricular function is the cornerstone of risk stratification with respect to long-term prognosis after an acute coronary syndrome (ACS) [3]. However, foreseeing re-infarctions in a specific patient is still elusive.

Vulnerable plaque is considered the critical point in the chain of events that provoke ACS and sudden cardiac death [4]. Recognizing the culprit lesion in nontransmural infarctions is not feasible in many cases, even by coronary angiography [5]. Numerous inflammatory markers, including highsensitivity CRP $[6,7]$ and a plethora of molecules have been evaluated as novel biomarkers; however, their predictive clinical utility is modest $[8,9]$.

Recently, many invasive and noninvasive diagnostic imaging modalities have been studied for identifying vulnerable plaques. We are now aware that even if ACS is usually provoked by the erosion or rupture of an atherosclerotic plaque, most plaque ruptures heal without evolving to a clinically relevant ACS $[4,5]$.

A variety of contributing factors beyond the identification of vulnerable plaques need to be integrated in order to predict and prevent ACS. Understanding the molecular mechanisms behind MI can lead to the prediction of this phenotype in asymptomatic individuals or post-MI patients [10].

An ACS occurring in an individual patient could be an interaction between inflammation, environmental factors and genetic predisposition [10]. The inflammatory process has been the better studied so far, while environmental factors such as smoking or arterial hypertension can be modified to a certain extent. However, the genetic code, which obviously plays a significant role in this interplay, needs better understanding [10]. The scientific field of clinical genomics is still in its infancy, since genome data are still scarce and even by proving new insights into genome structure and function, large multicenter studies will be needed to be carried out in order to

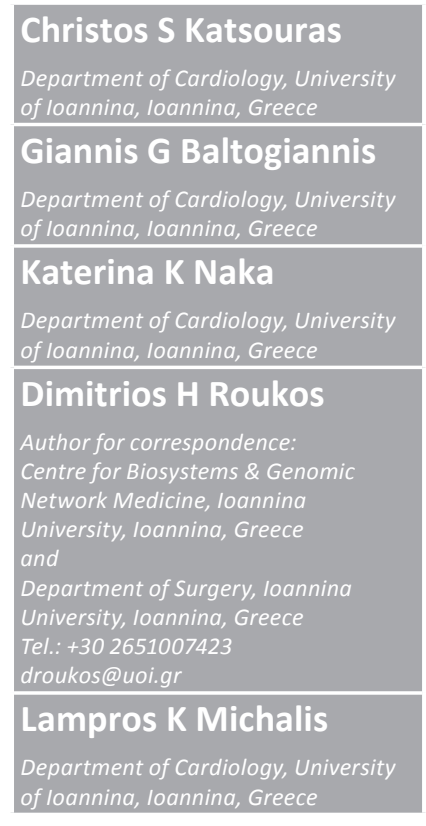

Future
Medicine 
test reproducibility and reliability of genome information.

\section{The prediction \& prevention paradox}

Although several modifiable risk factors have been identified, prevention of CAD remains a grand challenge. The prevention paradox is that a larger proportion of clinical events occur among the average majority than among the high-risk minority [11]. In fact, we should redefine the average-risk and high-risk subgroups in the population while considering the molecular characterization of the disease and understanding the highly complex genome-phenotype relationship.

Due to this prevention paradox, the goal of the 'Million Hearts' Initiative, which targets the 'high-risk' subpopulation and aims to reduce heart attacks and strokes by 1 million over the next 5 years in the USA [12], might be ambitious. In brief, according to the Million Hearts initiative, established risk factors are considered to be smoking, high levels of low-density lipoprotein cholesterol (LDL-C) and total cholesterol, hypertension, obesity and dysfunctional glucose metabolism, while its concept [12] is simple as it aims towards the prevention of CAD by smoking cessation and regulation of cholesterol, hypertension and diabetes (if it exists) with diet, physical activity and drugs [12]. We should wait to see the true effect of the prevention paradox. Further efforts in the genetics and genomics field will be needed to define the true high-risk profile of a subpopulation for future CAD events prevention. Despite enormous effort and major funding, there is no established method to predict with high accuracy acute cardiovascular ischemic events in individuals or groups. Noninvasive visualization of the total coronary tree by using computed tomography coronary angiography and identification of its pathological changes in both symptomatic and asymptomatic individuals is part of the efforts being made in order to predict MI before it occurs. However, the positive prediction value of such a method is not sufficiently high, thus not allowing its clinical application [13].

\section{Genetics \& high-throughput sequencing}

Human genome sequences, genetic variants and somatic mutations, molecular mechanisms and deeper insights into how the genome works in health and disease are now being considered the most promising biomedical research fields for understanding, predicting, preventing and curing common diseases including CAD events.
With the completion of the human genome sequence by the Human Genome Project in early 2000 and the follow-on HapMap project that created the reference database of human SNPs, high-throughput (HT) array technologies were developed for studying the role of SNPs in health and disease. GWAS to evaluate the associations between genetic variants and traits or diseases such as blood levels of lipids and CAD, or cancer, diabetes and other common disorders have become very popular over the last 5-6 years. Several arrays have allowed for efficient typing of hundreds of thousands to millions of SNPs across the genome. For example, the most recent collaboration relating genetic variants to blood lipids included 46 cohorts and more than 100,000 individuals, and an ongoing collaboration (CARDIOGRAM60) includes over 80,000 participants. These GWAS have dramatically increased the catalog of associations between SNPs and complex human diseases and other complex traits. However, the consensus of all these studies shows that the associated SNPs exert a small effect size explaining only $15-25 \%$ of heritability. This missing heritability has also been confirmed in a recent meta-analysis of several GWAS studying the correlation between SNPs and blood levels of lipids [14]. This analysis identified 95 loci associated with the level of one of total cholesterol, LDL-C, triglyceride and high-density lipoprotein $\mathrm{C}$ [14].

However, these common SNPs have a modest influence on disease risk and limited clinical utility as prognostic genetic tests of CAD events. In contrast with these common variants of the small effect, the rare variant of large effect might explain missing heritability and increase clinical utility. Such a paradigm of the rare variant of large effect concept represents familial hypercholesterolemia caused by mutations in the genes for the LDL-C receptor, among others. However, familial hypercholesterolemia accounts for only $5-10 \%$ of CAD events among individuals younger than 45 years of age. As familial hypercholesterolemia substantially increases the risk of coronaryrelated mortality as compared with the general population, genetic testing at a young age with early and intensive lipid-lowering treatment in mutation carriers can benefit these individuals [11].

\section{Postzygotic mosaicism}

In clinical medicine, the majority of patients with multifactorial disease such as CVD, diabetes or cancer have no significant family history, suggesting that in the majority of cases common disorders are sporadic. Thus, the limited clinical 
utility of GWAS is not surprising. In a recent review of three of the latest papers [15], evidence is provided that postzygotic changes, particularly copy numbers aberrations (CNAs), have an important causative role in the development of human diseases [15]. If this is true, and given that postzygotic or somatic mutations (also termed as somatic mosaicism) are aberrations acquired during the lifetime, new genomics studies and changes in biobanking sampling will be required [15]. The new biobanking strategy includes collection of several samples at different times from the same individual to assess the dynamics of genome alterations derived through time. That means one single sampling from an individual does not provide comprehensive genomic information. As common diseases occur at various ages depending on the accumulation of somatic aberration (probably over a specific score for each individual's mutations), multiple sampling and genomics analyses will be required to predict disease manifestation $[15,16]$.

\section{HT technologies}

Somatic mosaicism and particularly the postzygotic CNAs can be identified rapidly, cheaply and accurately using NGS. With plummeting costs for genome sequencing and evidence for the importance of somatically-acquired CNAs and other classes of mutations in etiopathogenesis of common diseases [15-17], new somatic sequencing studies for CVD are expected since cost-effective NGS-based analysis is warranted.

It is unclear whether all classes of mutations, for example point mutations and larger structural changes as well as inherited variants and somatic alterations, captured by future studies using a variety of NGS and microarray platforms will lead to robust comprehensive diagnostic and prognostic tests. It is probable that beyond wholegenome sequencing/whole-exome sequencing, further exploration of biological samples from patients with CVD will be required to relate causal mutations with gene and genome function and collectively with disease-specific phenotypes. These genome-wide mapping platforms coupled with integrated bioinformatics will provide insights into gene expression regulation. For example, RNA sequencing provides valuable information on transcripts, noncoding RNAs, post-transcriptional mutations and gene fusions. Subsequently, chromatin immunoprecipitation followed by HT sequencing can be used for mapping the genomic location of transcription factor binding and histone modifications in living cells. This mapping of sequence-specific transcription factor binding sites across the genome is crucial for exploring regulatory networks that underlie cellular function in health and disease [18]. Since current evidence suggests that most disease-associated variants lie, in the vast majority ( 93\%), within the noncoding sequences [18], it is essential to also study the noncoding long RNAs and short miRNAs to get a comprehensive view of functional regulatory networks that control gene expression in disease states [19-24].

Emerging advances in the genome sequence catalog for each common disease coupled with increasing understanding of functional regulation of the human genome in health and disease will provide the genomic basis of disease. By applying modern sequencing and microarray technologies in biological samples, and analyzing whole-genome sequencing/whole-exome sequencing, RNA sequencing, chromatin immunoprecipitation sequencing and array multilayer data, and by relating this integrated genome architecturederived information to clinical data for individual patients, we will begin to enter into the clinical genomics era. This progress paves the way towards one of most promising and stochastic areas of clinical genomics and biology: the goal to reach the ambitious destination of personalized cardiology.

\section{Financial \& competing interests disclosure}

The authors have no relevant affiliations or financial involvement with any organization or entity with a financial interest in or financial conflict with the subject matter or materials discussed in the manuscript. This includes employment, consultancies, honoraria, stock ownership or options, expert testimony, grants or patents received or pending, or royalties.

No writing assistance was utilized in the production of this manuscript.

\section{References}

1 Steg PG, James SK, Atar D et al.; The Task Force on the management of ST-segment elevation acute myocardial infarction of the European Society of Cardiology. ESC Guidelines for the management of acute myocardial infarction in patients presenting with ST-segment elevation. Eur. Heart J. 33, 2569-2619 (2012).

2 Thygesen K, Alpert JS, Jaffe AS, Simoons ML, Chaitment BR, White HD. Third universal definition of myocardial infarction. Eur. Heart J. 33, 2551-2567 (2012).
The Task Force for the Diagnosis and Treatment of Acute and Chronic Heart Failure 2012 of the European Society of Cardiology. Developed in collaboration with the Heart Failure Association (HFA) of the ESC. ESC Guidelines for the diagnosis and treatment of acute and chronic heart failure. Eur. Heart J. 33, 1787-1847 (2012). 
4 Arbab-Zadeh A, Nakano M, Virmani R et al. Acute coronary events. Circulation 125, 1147-1156 (2012).

5 Sharif F, Murphy RT. Current status of vulnerable plaque detection. Catheter. Cardiovasc. Interv. 75, 135-144 (2010).

6 Schnabel R, Lubos E, Rupprecht HJ et al. B-type natriuretic peptide and the risk of cardiovascular events and death in patients with stable angina results from the atherogene study. JACC 47(3), 552-558 (2006).

7 Saunders JT, Nambi V, de Lemos JA et al. Cardiac troponin $\mathrm{T}$ measured by a highly sensitive assay predicts coronary heart disease, heart failure, and mortality in the Atherosclerosis Risk in Communities Study. Circulation 123, 1367-1376 (2011).

8 Siasos G, Tousoulis D, Athanasiou D et al. Novel risk factors related to stable angina. Curr. Pharm. Des. 19(9), 1550-1561 (2013).

9 Kaski JC, Fernandez-Berques DJ, ConsueqraSanchez L et al. A comparative study of biomarkers for risk prediction in acute coronary syndrome-results of the SIESTA (Systemic Inflammation Evaluation in non-ST-elevation Acute Coronary Syndrome) study. Atherosclerosis 212, 636-643 (2010).

10 Anwaruddin S, Askari AT, Topol EJ. Redefining risk in acute coronary syndromes using molecular medicine. JACC 49(3), 279-289 (2007).
11 Holmes MV, Harrison S, Talmud PJ et al. Utility of genetic determinants of lipids and cardiovascular events in assessing risk. Nat. Rev. Cardiol. 8(4), 207-221 (2011).

12 Frieden TR, Berwick DM. The 'Million Hearts' initiative - preventing heart attacks and strokes. N. Engl. J. Med. 365(13), e27 (2011).

13 Kihara Y. After the triumph of cardiovascular medicine over acute myocardial infarction at the end of the 20th Century can we predict the onset of acute coronary syndrome? (Con)-. Circ. J. 75(8), 2019-2026 (2011).

14 Teslovich TM, Musunuru K, Smith AV et al. Biological, clinical and population relevance of 95 loci for blood lipids. Nature 466, 707-713 (2010).

15 Forsberg LA, Absher D, Dumanski JP. Non-heritable genetics of human disease: spotlight on postzygotic genetic variation acquired during lifetime. J. Med. Genet. 50(1), 1-10 (2013).

16 Chen R, Mias GI, Li-Pook-Than J et al. Personal omics profiling reveals dynamic molecular and medical phenotypes. Cell 148, 1293-1307 (2012).

17 Abyzov A, Mariani J, Palejev D et al. Somatic copy number mosaicism in human skin revealed by induced pluripotent stem cells. Nature 492(7429), 438-442 (2012).
18 Maurano MT, Humbert R, Rynes E et al. Systematic localization of common disease-associated variation in regulatory DNA. Science 337(6099), 1190-1195 (2012).

19 Stamatoyannopoulos JA. What does our genome encode? Genome Res. 22(9), 1602-1611 (2012).

20 Gerstein MB, Kundaje A, Hariharan M et al. Architecture of the human regulatory network derived from ENCODE data. Nature 489 (7414), 91-100 (2012).

21 Ideker T, Krogan NJ. Differential network biology. Mol. Syst. Biol. 8, 565 (2012).

22 Dunham I, Kundaje A, Aldred SF et al.; ENCODE Project Consortium. An integrated encyclopedia of DNA elements in the human genome. Nature 489(7414), 57-74 (2012).

23 Barabási AL, Gulbahce N, Loscalzo J. Network medicine: a network-based approach to human disease. Nat. Rev. Genet. 12, 56-68 (2011).

24 Erler JT, Linding R. Network medicine strikes a blow against breast cancer. Cell 149(4), 731-733 (2012).

\section{Website}

101 WHO. Cardiovascular diseases. Factsheet no. 317 (2012).

www.who.int/mediacentre/factsheets/fs317/ en/index.html 